\title{
CHIRAL STRING IN A CURVED SPACE: GRAVITATIONAL SELF-ACTION
}

\author{
Yu.V. GRATS *, A.A. ROSSIKHIN and A.O. SBOICHAKOV \\ Department of Theoretical Physics, M.V. Lomonosov Moscow State University \\ 119899, Moscow, Russia
}

\begin{abstract}
We analyze the effective action describing the linearised gravitational self-action for a classical superconducting string in a curved spacetime. It is shown that the divergent part of the effective action is equal to zero for the both Nambu-Goto string and chiral superconducting string.
\end{abstract}

Now it is well understood that topological defects in quantum field theory may play an essential role in cosmology, and the vertex line defects describable on a macroscopic scale as cosmic strings are most likely to be actually generated at phase transitions in the early Universe. ${ }^{1}$ Strings are predicted by many of the commonly considered field theoretical models, and their gravitational effects become the question of increasing interest during last twenty years. Indeed, the evolution of a cosmic string network may play an essential role in the formation of the large scale structure of the Universe observed at the present time. But possible cosmological applications is not the only reason for this consideration. There are some nontrivial features in the interaction of strings with gravity. One of them is the absence of gravitational bremsstrachlung from colliding straight gravitating segments ${ }^{2}$, while test particle freely moving near Nambu-Goto string emits gravitational waves. ${ }^{3}$ Another one is the absence of classical linearised self-interaction of a Nambu-Goto string with a gravitational field in a four-dimensional Minkowski spacetime. ${ }^{4,5}$ The last result is nontrivial. We know that in a lot of applications the radius of curvature of a string is much larger that its transverse diameter. So, the string can be considered to be infinitely thin, and the dynamics of the vertex line can be described with the use of the Nambu-Goto action or its generalization, if the interaction with an electromagnetic field or the fields of axion and dilaton is taken into account. In these cases underlying gauge theory is used only for the fixing the parameters in a string action. Interaction with matter fields give rise to outgoing flaxes of corresponding quanta.

*E-mail address: grats@string.phys.msu.su 
At the same time zero thickness of strings must lead to the divergence of a self-force. This problem is well known in classical electrodynamics. In the case of point charge in Minkowski space such a situation was considered by Dirac ${ }^{6}$ who showed that the divergence in the self-action can be removed by the renormalization of the particle mass with the use of corresponding classical counterterm. Analogous problem has been studied in a lot of papers for the string interacting with linearised gravitational field on Minkowski background, with electromagnetic field, with dilaton and axion, see $[4,5]$ and the Refs. therein. From our point of view, the absence of classical linearised gravitational self-interaction is the most interesting result of the previous consideration. In the case of straight Nambu-Goto string this conclusion is more or less obvious because Newtonian potential of such a string is equal to zero. But for the curved string, for the string in an external gravitational field or for the string with some internal degrees of freedom, say a currentcarrying one, the answer is not obvious. So, we can say that even at classical level interaction of cosmic strings with gravitational field, and in particular gravitational self-interaction, is not properly investigated.

Here we consider gravitational self-action of a chiral currentcarrying string, i.e. a superconducting string with an isotropic current. Chiral strings are of increasing interest because this simple model may be considered as a first step towards the understanding of a full theory including superconductivity in strings. ¿From the other hand, vertex defects of this type arise naturally in some kinds of supersymmetric theory, and there are some interesting cosmological consequences of their existence. In particular, current can stabilize cosmic string, and chiral vortons are expected to be more stable than non-chiral string loops.

We use the metric with the signature $(+---)$ and the system of units $c=1$.

In order to describe the macroscopic effects of the currents arising from the processes of superconducting kind, Witten ${ }^{7}$ proposed the use of simple generalization of the Nambu-Goto model which is characterized by the action

$$
S_{s t r}=\int d^{2} \zeta \sqrt{-\gamma}\left(-\mu+\frac{1}{2} \gamma^{a b} \partial_{a} \phi \partial_{b} \phi\right),
$$

where $\zeta^{a} a=0,1$ are the internal coordinates on the world sheet which is imbedded in the four-dimensional spacetime with coordinates $x^{\mu}$ and metric $g_{\mu \nu}, \gamma$ is the determinant of the induced metric $\gamma_{a b}=g_{\mu \nu} x_{, a}^{\mu} x_{, b}^{\nu}$.

In this model there is an additional internal scalar field $\phi$ in terms of which one can express the worldsheet supported conserved current

$$
\mathcal{J}^{a}=\frac{1}{\sqrt{-\gamma}} e^{a b} \phi_{, b} \quad \mathcal{J}^{a} ; a=0
$$

and energy-momentum tensor

$$
\mathcal{T}_{a b}=\mu \gamma_{a b}+\phi_{, a} \phi_{, b}-\frac{1}{2} \gamma_{a b} \phi_{, c} \phi^{, c}
$$


In Eq. (3) $e^{a b} / \sqrt{-\gamma}$ stands for the two-dimensional Levi-Civita tensor, with $e^{01}=$ $-e^{10}=-1$.

The surface current $\mathcal{J}^{a}$ and energy-momentum tensor $\mathcal{T}_{a b}$ are the two-dimensional tensorial fields defined on the worldsheet of a string, while corresponding fourdimensional sources which determine electromagnetic and gravitational interaction of the string read

$$
\begin{gathered}
J^{\mu}(x)=\int d^{2} \zeta \sqrt{-\gamma} \mathcal{J}^{a} \partial_{a} x^{\mu} \delta(x, x(\zeta)), \\
T^{\alpha \beta}(x)=\int d^{2} \zeta \sqrt{-\gamma} \mathcal{T}^{a b}(\zeta) \partial_{a} x^{\alpha} \partial_{b} x^{\beta} \delta^{4}(x, x(\zeta)) .
\end{gathered}
$$

And, as in the Dirac's case, the ultraviolet divergence in a self-force, if it is, is the consequence of a distributional nature of the currents (4).

Below we will consider the case of chiral string with a so-called null current. This condition does not mean that the current itself must be equal to zero, but that it is timelike

$$
\gamma_{a b} \mathcal{J}^{a} \mathcal{J}^{b}=0=\gamma^{a b} \phi_{, a} \phi_{, b} .
$$

For the chiral string the last term in Eq. (3) vanishes, but the energy-momentum tensor still differs from the one describing string of the Nambu-Goto type. So, one can suppose the existence of some differences in the interaction of a Nambu-Goto and currentcarrying strings with an external gravitational field.

To allow for the interaction with a linearised gravitational perturbations, one must perform the replacement $g \mapsto g+h$ which is equivalent to the augmentation of the string Lagrangian in Eg. (1) by the interaction term

$$
\mathcal{L}_{s t r}[g] \mapsto \mathcal{L}_{s t r}[g]-\frac{1}{2} \mathcal{T}^{a b} \partial_{a} x^{\mu} \partial_{b} x^{\nu} h_{\mu \nu}(x(\zeta)),
$$

As for the gravitational action, we must expand it in powers of $h_{\mu \nu}$ up to the second-order terms.

Corresponding equation for the linear metric perturbations has the wellknown form

$$
\delta \frac{\delta S_{g r}[g]}{\delta g^{\mu \nu}(x)}=-\frac{\sqrt{-g}}{2} T_{\mu \nu}(x) .
$$

Second-order variational derivative of the gravitational action can be found for example in Ref.[7].

Substituting the solution of the Eq. (7) back into the total action, we obtain

$$
\begin{gathered}
S_{t o t}[g, x, \phi]=S_{g r}[g]+S_{s t r}[g, x, \phi]+ \\
+\frac{G}{4} \int d^{2} \zeta \sqrt{-\gamma} \int d^{2} \zeta^{\prime} \sqrt{-\gamma^{\prime}} \mathcal{T}^{\mu \nu}(\zeta) \bar{G}_{\mu \nu \mu^{\prime} \nu^{\prime}}^{g r}\left(\zeta, \zeta^{\prime}\right) \mathcal{T}^{\mu^{\prime} \nu^{\prime}}\left(\zeta^{\prime}\right),
\end{gathered}
$$

where for simplisity we use the notation $\mathcal{T}^{\mu \nu}=\partial_{a} x^{\mu} \partial_{b} x^{\nu} \mathcal{T}^{a b}$.

The second and the third terms in the Eq.(8) depend on the string variables and describe the dynamics of an infinitely thin cosmic string which interacts with it's 
linearised gravitational field. When gravitational radiation from the string is not taken into account $\bar{G}_{\mu \nu \mu^{\prime} \nu^{\prime}}^{g r}$ is the real part of the Feynman propagator.

In the Lorentz gauge, when $\left(h_{\mu \nu}-1 / 2 g_{\mu \nu} h\right)^{; \mu}=0$, this equation becomes

$$
\left(\delta_{\mu}^{\alpha} \delta_{\nu}^{\beta} \square+H_{g r \mu \nu}^{\alpha \beta}\right) G_{\alpha \beta \mu^{\prime} \nu^{\prime}}^{g r}\left(x, x^{\prime}\right)=-16 \pi\left(g_{\mu \mu^{\prime}} g_{\nu \nu^{\prime}}-\frac{1}{2} g_{\mu \nu} g_{\mu^{\prime} \nu^{\prime}}\right) \delta\left(x, x^{\prime}\right),
$$

and $H_{g r}^{\alpha \beta}{ }_{\mu \nu}$ takes the form

$$
H_{g r}^{\alpha \beta}{ }_{\mu \nu}=-2 R_{\mu \nu}^{\alpha \beta}-2 \delta_{(\mu}^{\alpha} R_{\nu)}^{\beta}-\frac{1}{2} g_{\mu \nu} g^{\alpha \beta} R+g_{\mu \nu} R^{\alpha \beta}+\delta_{\mu}^{\alpha} \delta_{\nu}^{\beta} R .
$$

Proceeding along the same line as in Ref. [9] one can obtain the expression

$$
\begin{aligned}
\bar{G}_{\mu \nu \mu^{\prime} \nu^{\prime}}^{g r}\left(x, x^{\prime}\right)= & 2 \Delta^{1 / 2}\left(x, x^{\prime}\right)\left(\epsilon_{\mu \mu^{\prime}} \epsilon_{\nu \nu^{\prime}}-\frac{1}{2} g_{\mu \nu} g_{\mu^{\prime} \nu^{\prime}}\right) \delta(\sigma)+ \\
& +\theta(-\sigma) v_{\mu \nu \mu^{\prime} \nu^{\prime}}\left(x, x^{\prime}\right) .
\end{aligned}
$$

In the last equation $\Delta\left(x, x^{\prime}\right)=-(-g(x))^{-1 / 2} \operatorname{det}\left(\partial_{\mu \nu^{\prime}}^{2} \sigma\left(x, x^{\prime}\right)\right)\left(-g\left(x^{\prime}\right)\right)^{-1 / 2}, \epsilon_{\mu \mu^{\prime}}$ is the bivector of parallel transport, $\sigma$ is the half of geodesic distance between points $x$ and $x^{\prime}$, and $v_{\mu \nu \mu^{\prime} \nu^{\prime}}\left(x, x^{\prime}\right)$ denotes some regular function, which must be calculated for any particular case.

¿From Eq. (11) we can see that the part of the action we would expect to be divergent is

$$
\begin{aligned}
S_{d i v}=\frac{G}{2} & \int d^{2} \zeta \sqrt{-\gamma(\zeta)} \int d^{2} \zeta^{\prime} \sqrt{-\gamma\left(\zeta^{\prime}\right)} \Delta^{1 / 2}\left(\zeta, \zeta^{\prime}\right) \delta\left(\sigma\left(\zeta, \zeta^{\prime}\right)\right) \times \\
& \times \mathcal{T}^{\mu \nu}(\zeta)\left(\epsilon_{\mu \mu^{\prime}} \epsilon_{\nu \nu^{\prime}}-\frac{1}{2} g_{\mu \nu} g_{\mu^{\prime} \nu^{\prime}}\right) \mathcal{T}^{\mu^{\prime} \nu^{\prime}}\left(\zeta^{\prime}\right)
\end{aligned}
$$

In four spacetime dimensions the integral in Eq.(12) diverges logarithmically as $\zeta \rightarrow \zeta^{\prime}$, and it must be renormalized. This integral can be estimated with the use of the normal Riemann coordinates on the world sheet with the origin at the point $\zeta$ or using any other regularization scheme, see for example Ref. [5],

$$
S_{\text {eff }}=2 G \log \frac{\triangle}{\delta} \int d^{2} \zeta \sqrt{-\gamma} \mathcal{T}^{\mu \nu}\left(g_{\mu \gamma} g_{\nu \delta}-\frac{1}{2} g_{\mu \nu} g_{\gamma \delta}\right) \mathcal{T}^{\gamma \delta}+S_{\text {fin }},
$$

where $\delta$ is a short-range cutoff length, which is identified with the string radius, while $\Delta$ must be introduced because of the logarithmic dependence of $\delta$ in Eq. (13). This infrared regularization length corresponds to the distance at which conical geometry of string is disturbed by the background geometry.

Now we have to substitute the explicit expression for $\mathcal{T}^{\mu \nu}$ into Eq. (13). We then obtain

$$
S_{\text {div }}=G \log \frac{\Delta}{\delta} \int d^{2} \zeta \sqrt{-\gamma} \mathcal{J}^{4}
$$


We calculated the divergent part of the effective action for a currentcarrying string interacting with gravitational field. Eq. (14) shows that linearised gravitational self-interaction in the presence of an external gravitational field is equal to zero for the both Nambu-Goto and superconducting chiral string. This result generalizes the one obtained in Refs. $[4,5]$.

The finite part of the action determines the effect of the topological self-action which is equal to zero in the Minkowski space. There is no general prescription for its calculation. Sometimes the finite part of the self-force can be calculated explicitly ${ }^{10,11}$ or with the use of perturbative technique. ${ }^{12}$

If $\mathcal{J}^{2} \neq 0$ the situation becomes some more interesting. In this case the logarithmic divergent term (14) does not renormalize the bare action (1) because there is no term $\sim \mathcal{J}^{4}$ in Eq. (1). It seems that this seems that the full noncontradictional theory of superconducting string must be a nonlinear theory.

\section{Acknowledgments}

This work was supported by the Russian Foundation for Basic Research, grant 99-02-16132.

\section{References}

1. The Formation and Evolution of Cosmic Strings, eds. G. Gibbons, S. Hawking, and T. Vachaspaty (Cambridge University Press, Cambridge, 1990).

2. D.V. Gal'tsov, Yu.V. Grats, and P.S. Letelier, Ann. of Phys. (NY) 224, 90 (1993).

3. A.N. Aliev and D.V. Gal'tsov, Ann. of Phys. (NY) 193, 142 (1989).

4. B. Carter and R.A. Battye, Phys. Lett. B430, 49 (1998).

5. A. Buonanno and T. Damour, Phys. Lett. B432, 51 (1998).

6. P.A.M. Dirac, Proc. Roy. Soc. A 167, 148 (1938).

7. B.S. DeWitt, Dynamical Theory of Groups and Fields (Gardon and Breach, New York, 1965).

8. E. Witten, Nucl. Phys. B249, 485 (1985).

9. B.S. DeWitt, R.W. Brehme, Ann. Phys. (NY) 9, 220 (1960).

10. Yu.V. Grats and A.A. Rossikhin, Theor. and Math. Phys. 123, 539 (2000).

11. E.R. Bezerra de Mello, V.B. Bezerra, and F. Moraes, Phys. Rev. D51, 7140 (1995).

12. D.V. Galt'sov, Yu.V. Grats, and A.A. Lavrent'ev, Phys. Atom. Nucl 58, 516 (1985). 